\title{
Percepciones sobre la discapacidad transmitidas por la prensa escrita española durante 1978, 1995 y 2007
}

\author{
Héctor Nauzet CuESTA SuÁREZ \\ cuestasuarez@gmail.com \\ Universidad de Las Palmas de Gran Canaria \\ María Rosa MARCHena Gómez \\ mmarchena@dedu.ulpgc.es \\ Universidad de Las Palmas de Gran Canaria \\ María Victoria AgUIAR PERERA \\ maguiar@dedu.ulpgc.es \\ Universidad de Las Palmas de Gran Canaria
}

Recibido 10 de junio de 2014

Aceptado: 16 de diciembre de 2014

\begin{abstract}
Resumen
Este trabajo pretende contrastar los modelos de percepción hacia la discapacidad más frecuentes a los que pertenecen los bloques informativos en determinados periódicos. Para llevarlo a cabo, hemos realizado un análisis de contenido de las unidades referenciales presentes en cualquier espacio informativo de los diarios $A B C$ y La Vanguardia durante varios meses de 1978, 1995 y 2007. En conclusión, podemos afirmar que es amplia la conquista del modelo tradicional -la discapacidad asociada a la exclusión- en 1978 y en 1995, acaparando la mitad o más de la mitad de las referencias. La influencia del modelo rehabilitador -discapacidad con planteamiento médico-especialista-, en cambio, es moderada. Y la repercusión del modelo social -reconocimiento social de sus derechos y autonomía- sigue siendo escasa, despuntando con claridad sólo en el $A B C$ de 2007.
\end{abstract}

Palabras clave: Discapacidad, periódico, modelo, análisis de contenido.

\section{Perceptions about Disability Transmitted by Spanish Written Press during 1978, 1995 and 2007}

\begin{abstract}
This paper aims to compare the most common belonging to the informative blocks that treat the issue of disability in certain newspapers. To accomplish this, we did a content analysis of referential units present in any information space of the daily $A B C$ and La Vanguardia for several months in 1978, 1995 and 2007. In conclusion, we can say that is wide conquest model traditionally -disability with the exclusionin 1978 and 1995, hogging half or more than half of the references. The influence of the rehabilitation model -disability with medical-specialist approach- is moderate instead. And the impact of the social model -social recognition of their rights and autonomy-is still scarce, clearly outperforming only in the $A B C$ of 2007.

Keywords: Disability, newspaper, model, content analysis.

\section{Referencia normalizada}

CUESTA SUÁREZ, Héctor Nauzet; MARCHENA GÓMEZ, María Rosa; y AGUIAR PERERA, María Victoria (2015): "Percepciones sobre la discapacidad transmitidas por la prensa escrita española durante 1978, 1995 y 2007”. Estudios sobre el Mensaje Periodístico. Vol. 21, Núm. 1 (enero-junio), págs.: 269282. Madrid, Servicio de Publicaciones de la Universidad Complutense.
\end{abstract}


Sumario: 1. Introducción; 1.1. Principales modelos socioconceptuales sobre discapacidad; 1.2. Discapacidad y medios de comunicación. 2. Metodología; 2.1. Categorización de las unidades referenciales; 2.2. Fiabilidad del instrumento categorizador. 3. Resultados y discusión; 3.1. Resultados obtenidos; 3.2. Discusión de los hallazgos. 4. Conclusiones. 5. Referencias bibliográficas.

\section{Introducción}

Este trabajo parte de un propósito inicial basado en plasmar una visión general y comparativa de los modelos socioconceptuales sobre discapacidad más frecuentes a los que pertenece el total de las unidades referenciales que traten el tema de la discapacidad, tomadas de los periódicos $A B C$ y La Vanguardia" durante los años 1978, 1995 y 2007.

\subsection{Principales modelos socioconceptuales sobre discapacidad}

Entre los modelos histórico-evolutivos planteados en esta última década, destaca en primer lugar la recopilación realizada por Schalock (2003) sobre investigaciones epistemológicas del concepto de discapacidad llevadas a cabo por autores como Goodey y Stainton (2001), entre otros. Y concluye exponiendo la existencia de tres modelos históricos de discapacidad:

- Modelo filosófico y teológico, que abarca del siglo I al siglo XVII, es un modelo aferrado a la razón como principio ideológico. En este modelo, las personas que carecían de raciocinio se consideraban degeneradas y carecían de todo tipo de derechos.

- Modelo biosocial, dominante desde el siglo XVII hasta la primera mitad del siglo XX, que considera a la discapacidad como un problema biológico e inherente a la persona. Por tanto, el individuo con discapacidad es el único causante de su situación. Por tanto, debe ser tratado y ayudado, pero sólo por profesionales expresamente cualificados.

- Modelo social-ecológico, que va de finales del siglo XX a principios del siglo XXI, donde se concibe la discapacidad como un asunto que nos concierne a todos, por lo que todo el entorno sociocultural debe implicarse y actuar al respecto.

A partir de esta clasificación inicial, en las dos últimas décadas, se han ido configurando diferentes modelos o formas sociales de ver la discapacidad. Y aunque unos paradigmas dominan sobre otros en los enfoques más progresistas, todos ellos no son mutuamente excluyentes, sino que conviven a lo largo del tiempo, pudiendo agruparse todas estas clasificaciones bajo tres grandes modelos:

- Modelo tradicional, que incluye todas las visiones de la discapacidad basadas en la exclusión, eliminación y ridiculización de estas personas, en la búsqueda de un origen fantástico-religioso a esta problemática y en la percepción de este colectivo como una carga familiar y social, entre otros aspectos.

- Modelo rehabilitador, que supone la percepción de la discapacidad desde un planteamiento médico-especialista, creando para estas personas centros e instituciones especializadas, y creyendo firmemente que la normalización se consigue a través de la segregación y el ocultamiento de la problemática (haciendo como si la discapacidad no existiera). 
- Modelo social, modelo en el que la persona con discapacidad es valorada socialmente, ya que se considera que tiene unos derechos y sus aportaciones son importantes en el entramado socioambiental en el que se desenvuelve. Dentro de este postulado, incluimos la visión bio-psico-social que está emergiendo actualmente.

\subsection{Discapacidad y medios de comunicación}

Existen muy pocos estudios hasta el momento que vinculan la discapacidad con el análisis periodístico. Y los que lo han hecho, se han centrado en prensa inglesa. Así por ejemplo, contamos con el informe elaborado por Keller et al. (1990) que estudia la discapacidad en diferentes periódicos americanos. A este trabajo, se suma la investigación comparativa realizada por Catlett y Martin (1993) que contrasta artículos de The Houston Post con informaciones de The Los Angeles Times, durante dos años. Y una de las más destacadas por su corte intercontinental, es la puesta en marcha por Auslander y Gold (1999), cuyo método contrasta periódicos canadienses con diarios israelíes.

Al margen de estos datos, hay que decir que en España también se anotan algunos trabajos al respecto, pero fundamentados todos ellos en prensa de tirada reducida. En este sentido, despuntan los estudios llevados a cabo por Aguado Díaz y Alcedo Rodríguez (1991) que plantean un análisis de este grupo humano a raíz de rotativos asturianos. Egea, Miras y Ripoll (1995) constatan otra investigación sobre la discapacidad vista desde la prensa murciana. Se añaden a estas indagaciones los trabajos de Díaz Aledo (2006), abordando esta misma temática, pero de nuevo desde prensa de tirada local y regional.

Todo ello apunta a que este trabajo lleva implícita una doble intencionalidad. Por un lado, busca recoger y conciliar los parámetros teóricos y metodológicos sembrados por todos estos informes, entre otros. Y por otro, aunarlos y trasladarlos a un terreno hasta ahora inexplorado: la prensa española de gran alcance.

Por otra parte, esta investigación se mueve también en el terreno social. En este ámbito, las personas con discapacidad son miradas con recelo por el entorno en el que se desenvuelven, debido en gran parte al desconocimiento y a la presencia de viejos estereotipos y prejuicios que acechan al colectivo desde prácticamente todos los ámbitos. Y los medios de comunicación, el periódico en este caso, constituyen una herramienta muy potente con la que romper con todas esas ideas preconcebidas. De hecho, en palabras de Villanueva et al. (2006), son fundamentales para hacer visibles a las minorías, dar a conocer sus problemas, reivindicar sus necesidades y fomentar su integración en la sociedad.

Sin embargo, si la comunicación de masas es mal empleada, se consigue el efecto contrario. Es decir, se logran arraigar aún más en el entramado sociocultural, los antiguos tópicos atribuidos tradicionalmente a estas personas. En palabras de Bueno Abad (1996), lo transmitido a través de estos medios atañe directamente a los procesos socializadores de los individuos, inculcando valores que remodelan antiguos de interpretación de la realidad, así como proponen otros nuevos.

A afirmaciones como ésta, se unen las del profesor Tajfel (1981), que asegura que en la configuración de actitudes sociales por el efecto mediático, se favorece la movilización de determinados sujetos o sectores, en pro de una reorganización sociocul- 
tural. Akoun (1998), Bourdieu (1996) y Trowler (1996) refuerzan estas bases ideológicas demostrando que los medios juegan un papel esencial en la creación de constructos sociales, en la implantación y refuerzo de las normas y en el desarrollo de actitudes de respeto y ayuda hacia los demás.

Hay otro factor remarcado desde estudios como el de Jaúregui Bereciartu (1990), que influye decisivamente en este binomio medio-sociedad. Se trata de la continua búsqueda de homogeneidad en la información que se transmite, hecho por el que la figura del lector se ve instrumentalizada. Por lo tanto, el poder aglutinante de los medios de comunicación, referidos en este caso al sector periodístico, hace de nuevo acto de presencia.

\section{Metodología}

\subsection{Categorización de las unidades referenciales}

Para llevar a cabo este trabajo, se realizó un análisis de contenido de las unidades referenciales presentes en cualquier bloque informativo de los ejemplares de los diarios $A B C$ y La Vanguardia. La longevidad y amplia tirada de estos periódicos y, en consecuencia, el tiempo de potencial interacción e influencia en el contexto social próximo, fueron las principales causas que nos llevaron a seleccionar estos casos de estudio. Se analizaron los años 1978 (etapa previa a la publicación de la Ley de Integración Social del Minusválido, LISMI), 1995 (la integración escolar y el conocimiento de la LISMI es una realidad social) y el 2007 (existen avances sociales y europeos importantes en las normativas que apoyan la discapacidad). El segmento temporal de estos años fue el siguiente:

- Año 1978: Ejemplares de ABC de abril, mayo y junio. Ejemplares de La Vanguardia de enero, febrero y marzo.

- Año 1995: Ejemplares de ABC de octubre, noviembre y diciembre. Ejemplares de La Vanguardia de julio, agosto y septiembre.

- Año 2007: Ejemplares de ABC de enero, febrero y marzo. Ejemplares de La Vanguardia de octubre, noviembre y diciembre.

Según Krippendorff (1990), las unidades referenciales se definen a partir de determinados objetos, sucesos, actos o ideas. En nuestro caso, estas unidades hacen alusión a una serie de categorías vinculadas a los modelos sobre discapacidad ya definidos, y que fueron conformándose a medida que avanzaba la investigación y se iban estudiando las siguientes unidades de análisis específicas:

- El lenguaje textual: titular, lead, texto periodístico, entradilla, ventanas y ladillos.

- El lenguaje icónico: código cromático de la foto, su luminosidad, la escala de planos, el ángulo de toma y la extensión.

- El emplazamiento: ubicación del número de página, situación en la página y extensión.

El número total de categorías resultantes fue de veinticinco, generadas bajo una estrategia mixta, es decir, tanto de modo deductivo como inductivo. Todas ellas se agruparon en los siguientes modelos teóricos: 
- Modelo tradicional: Vida innecesaria. Relación con la fantasía y la religión. Carga para la familia. Carga para la sociedad. Exaltación de la discapacidad como tragedia personal. Subestimación de capacidades. Mundo aparte y desconocido. Objeto de ridiculización y desprecio social. Relación con la delincuencia y el vandalismo. Víctima de una agresión física. Segregación social mal asistida.

- Modelo rehabilitador: Segregación en lugares ideales. El tratamiento médicoespecialista como principal solución. La discapacidad como etiqueta personal y/o negativa. Ocultamiento injustificado de la persona. Crítica a las acciones integradoras.

- Modelo social: Vida independiente y normalizada. Superación personal. Cumplimiento de derechos. Crítica a la terminología tradicional. Existencia útil. La sociedad como agente de cambio. La discapacidad como rasgo independiente de la persona. Valoración de las capacidades. Entorno accesible.

\subsection{Fiabilidad del instrumento categorizador}

Una vez confeccionado y ensayado el instrumento por el que clasificamos una parte de las unidades referenciales en base a las categorías ya mencionadas, la necesidad de elaborar inferencias reproducibles y válidas en el análisis de contenido, exigió demostrar su nivel de fiabilidad (Cea D'Ancona, 1996). Krippendorff (1990) remarca que la fiabilidad de los datos ha de cumplir los siguientes requisitos:

- Estabilidad. Es el grado en el que permanecen invariantes los resultados de la clasificación a lo largo del tiempo.

- Reproductibilidad. Es el grado en que pueden alcanzarse los mismos resultados en circunstancias diferentes.

- Exactitud. Es el grado en el que se ajusta funcionalmente a un criterio estándar o norma. Como rara vez se establecen codificaciones estándar, este último criterio de fiabilidad apenas se aplica.

Para verificar que se dan estas premisas, han de arbitrarse unas reglas de codificación que permitan que distintos codificadores o jueces alcancen, independientemente unos de otros, los mismos resultados (Cea D'Ancona, 1996). A este respecto, Andréu (2000) se pronuncia afirmando que la fiabilidad surge del acuerdo alcanzado entre estos jueces sobre la asignación de las unidades a las diversas categorías.

Para lograr ese posible acuerdo, Krippendorff (1990) establece que los jueces deben estar familiarizados con el material a registrar, pero además deben ser capaces de manejar con cierta destreza las categorías y términos que componen el lenguaje de datos. Atendiendo a estas finalidades, convocamos a un grupo formado por 4 jueces relacionados con la discapacidad desde diferentes entornos.

Una vez seleccionados, se les instruye y entrena en el proceso de registro. Para ello, se procede a la lectura del libro de códigos y a la aplicación del instrumento empleado en una pequeña submuestra, simplemente para comprobar que lo han entendido. La coincidencia lograda en este primer ensayo es casi absoluta.

Al mes siguiente, se procede a la verificación de la fiabilidad del instrumento por parte de cada juez en solitario. Según Krippendorff (1990), en esta parte de la verifi- 
cación, los individuos deben poder operar con las instrucciones de registro como única guía; y con un grado absolutamente mínimo de comunicación informal entre ellos, para que no surjan acuerdos subrepticios sobre sus propias interpretaciones. Respetando estos preludios, le hacemos llegar a cada uno por correo electrónico de nuevo el libro de códigos, junto a otra submuestra de fragmentos textuales e imágenes a categorizar. La cumplimentación de estos registros la recibimos sin problemas al mes siguiente.

En esta ocasión, para la baremación estadística de sus niveles de coincidencia, todos los datos obtenidos se introdujeron en una base de datos Statistical Package for the Social Sciences (SPSS) para Windows, versión 15.0. Para ello, se diseñó previamente una tabla en la que se volcaron el número de coincidencias existentes entre cada uno de los jueces y el investigador principal.

A continuación, se calculó el índice Kappa k como medida de concordancia entre observadores. El índice de acuerdo Kappa $\mathrm{k}$ proporciona una medida del grado de acuerdo existente entre dos jueces al evaluar una serie de sujetos u objetos (Cohen, 1960). Los valores del índice Kappa $\mathrm{k}$ oscilan entre 0 y 1, considerándose que existe un mayor grado de concordancia cuanto mayor sea dicho índice.

Partiendo de todas estas directrices y de los modelos socioconceptuales sobre discapacidad ya definidos, el índice Kappa $\mathrm{k}$ es de 0,$91 ; 0,61 ; 0,95$ y 0,86 para los jueces $1,2,3$ y 4 respectivamente. Ante esto, se pudo afirmar que el nivel de coincidencias de los jueces en relación al investigador principal fue bastante alto, por lo que el instrumento empleado resultó fiable.

\section{Resultados y discusión}

\subsection{Resultados obtenidos}

Si nos detenemos en las frecuencias categoriales que aluden al modelo tradicional, vemos la predominancia en casi todos los años de la categoría "Exaltación de la discapacidad como tragedia personal". Los únicos años en que no lideran estas frecuencias son 1978 de $A B C$ y 2007 de La Vanguardia. Aun así, quedan en esos años en segundo lugar, sólo superados por "Carga para la familia" $(25.61 \%$ frente a $18.69 \%)$ y "Segregación social mal asistida" $(24.14 \%$ frente a $22.66 \%)$ respectivamente en cada caso. Para comprobar todo esto, le remitimos a la gráfica 1.

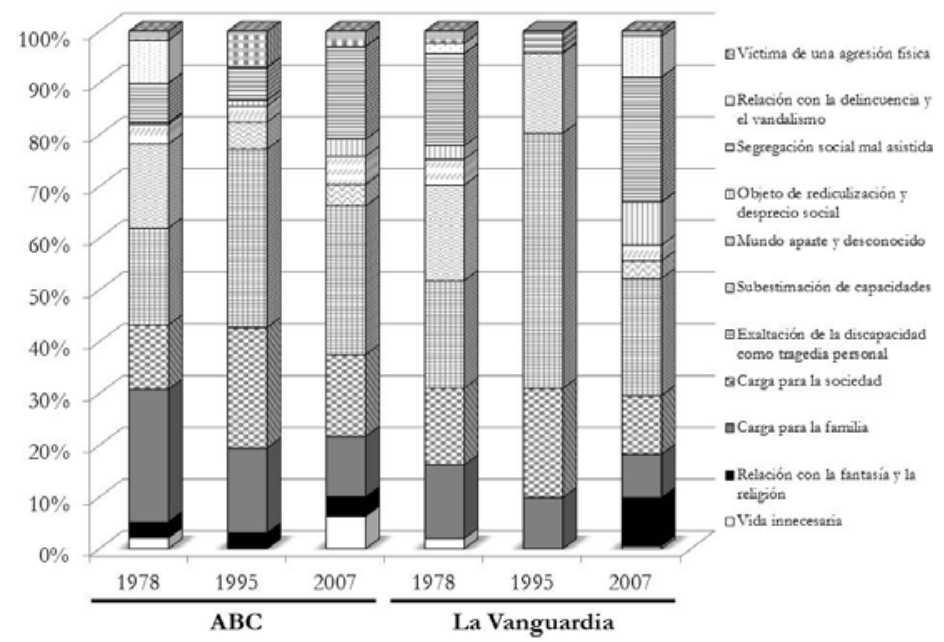

Gráfica 1: Porcentajes de las unidades presentes en cada categoría del Modelo Tradicional 
Por su parte, en el modelo rehabilitador predomina rotundamente la "Segregación en lugares ideales", rondando en el año 78 de ambos diarios el $80 \%$ del total de las referencias a ese modelo. La gráfica 2 da cuenta de estas informaciones.

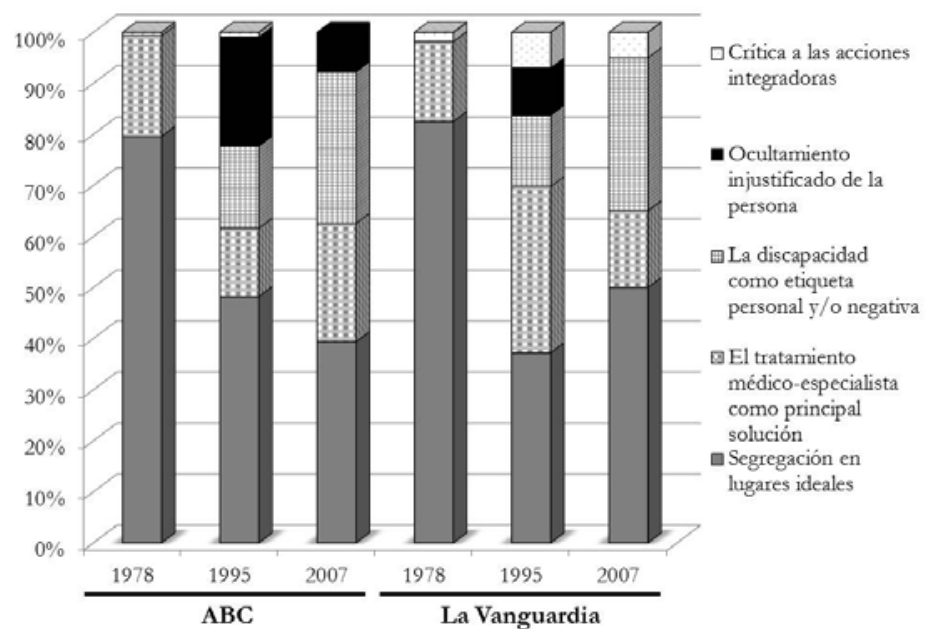

Gráfica 2: Porcentajes de las unidades presentes en cada categoría del Modelo Rehabilitador

En cambio, el modelo social muestra preferencia frecuencial por la categoría "La sociedad como agente de cambio" en todos los años analizados de $A B C$. El porcentaje de esta categoría en La Vanguardia también es alto. Sin embargo, en todos los casos ocupa la segunda posición, erigiéndose como dominantes "Cumplimiento de derechos" en 1978 y 2007; y "Entorno accesible" en 1995. Se atestiguan estos datos en la gráfica 3.

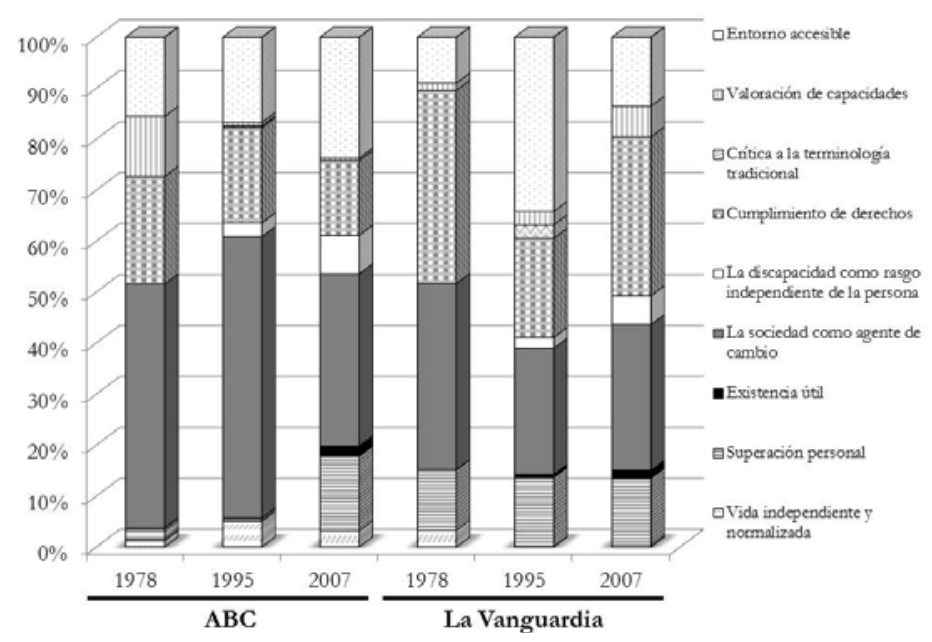

Gráfica 3: Porcentajes de las unidades presentes en cada categoría del Modelo Social 
Y a modo de síntesis infográfica, mostramos los totales porcentuales de cada modelo por año de estudio. En base a estas consideraciones, percibimos el dominio del modelo tradicional en ambos rotativos durante 1978 y 1995, acaparando en el 78 más de la mitad de la muestra (56.95\% y 56.04\%); y en el 95, casi la mitad $(48.09 \%$ y $48.53 \%$ ). El único año en que destaca el modelo social es el 2007 de $A B C$ con un $43.13 \%$; ya que en La Vanguardia su dominio dista ligeramente del modelo tradicional. Hablamos de un $39.46 \%$ de presencia del modelo social frente a un $38.38 \%$ de modelo tradicional en este diario durante 2007. Todo ello lo reflejamos en la gráfica 4.

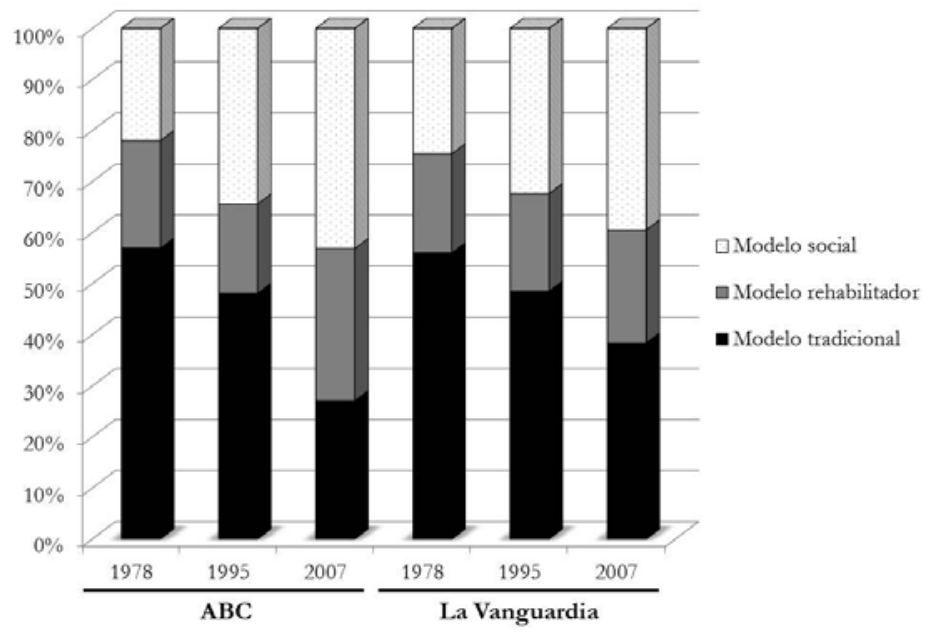

Gráfica 4: Porcentajes del total de categorías que hay por cada modelo

\subsection{Discusión de los hallazgos}

En primer lugar, en el reparto de todas las unidades referenciales ya nombradas entre las diferentes categorías del modelo tradicional, queda patente la hegemonía frecuencial de la "Exaltación de la discapacidad como tragedia personal" en casi todos los trimestres. Esto coincide directamente con la imagen merecedora de pena que se resalta de estas personas en estudios como el de Catlett y Martin (1993). Todo este tipo de representaciones sobre la discapacidad tiene su origen en el modelo filosófico y teleológico apuntado por Schalock (2003) y Goodey y Stainton (2001). Estos planteamientos se referían a la discapacidad como un eterno lastre para sus propios padres o para la sociedad, por lo que las personas que la tienen debían ser eliminadas o apartadas del entorno social. Todos ellos asociaban la discapacidad con el mal y la desgracia, despertando sentimientos de pena y lástima (Vega y Martín, 1999).

A pesar de esta predominancia, los años 1978 de $A B C$ y 2007 de La Vanguardia, esta categoría dominante es rebasada en frecuencia por "Carga para la familia" y "Segregación social mal asistida", respectivamente. Esto recuerda al corte marginal por el que son percibidas estas personas por la prensa, según el estudio de Del Río (1986). 
Todos estos elementos son la herencia que aún conservamos de la etapa de la percepción trágica de la discapacidad, época predominante hasta 1939, donde el colectivo era expuesto sistemáticamente por la prensa como víctima o verdugo de cualquier relato dramático (Martín Herrera, 2006).

En los hallazgos alusivos al modelo rehabilitador, por su parte, es claro el dominio tanto en frecuencias como en porcentajes, de la categoría "Segregación en lugares ideales". Ello se relaciona fundamentalmente con el modelo biosocial de Goodey y Stanton (2001), entre otros. La línea de todos estos postulados se orientaba hacia la institucionalización del colectivo, siendo tratado por profesionales especializados, únicos agentes capacitados para dar una respuesta.

Se observa, por tanto, el legado de la perspectiva rehabilitadora, dominante hasta los 70, y comentada por autores como Jiménez Lara (1999). De hecho, las frecuencias donde esta categoría encuentra su apogeo se ubican en el 78 de ambas gacetas.

Mediante las categorías de mayor presencia dentro del modelo social, percibimos una clara supremacía de "La sociedad como agente de cambio" frente al resto de categorías, durante todos los trimestre de $A B C$. La supremacía de esta categoría conecta con los estudios como el de Weinberg y Williams (1998). También indica que al menos en este periódico, la visión que impera sobre este modelo es la misma que transmitía el modelo social-ecológico tratado por Schalock (2003), entre otros. Todas estas posturas teóricas hacían hincapié en la extensión del asunto de la discapacidad del individuo al entramado social en el que éste se desenvuelve; por lo que su inclusión depende de él, pero también de las facilidades que ese contexto le permita.

A todo esto hay que añadir que esta categoría alcanza su punto álgido en 1995, dato que puede venir influenciado por normativas como la Ley 13/1982 de 7 de Abril, de Integración Social de los Minusválidos (LISMI). Ello se debe a que esta ley ya prescribía que para lograr la integración de este grupo social, tendría que participar el Estado, las comunidades autónomas, las corporaciones locales, los sindicatos, las entidades y organismos públicos, así como las asociaciones y personas privadas (Batanaz, 2000).

Si nos fijamos ahora en las categorías del modelo social predominantes en La Vanguardia, vemos cómo la de "Cumplimiento de derechos" se convierte en la más referenciada durante 1978 y 2007. Este hecho guarda parecido con lo expresado en el paradigma de Amate (2007) sobre la inclusión de los derechos humanos. A través de este tipo de esquemas ideológicos, se describe a la persona con discapacidad como alguien oprimido al que hay que ayudar en su defensa de derechos para el logro de una vida lo más independiente y normalizada posible.

En esta labor reivindicativa, tampoco hay que olvidar la influencia de importantes organismos, como es el caso de Comité Español de Representantes de Personas con Discapacidad (CERMI) o la Organización Nacional de Ciegos Españoles (ONCE), entre otras. A esto se le suman análisis, como el que realizan Marchena et al. (2007) sobre las vías para desarrollar en el colectivo rendimientos laborales sostenibles; así como el esfuerzo informador que ejercen día a día webs como "Down21", "Discapnet" o "Minusval2000".

Por otra parte, la frecuencia más contabilizada en La Vanguardia durante 1995 no se corresponde con ninguna de las categorías anteriores, sino con la de "Entorno ac- 
cesible". Esta supremacía categorial encuentra su origen en modelos como el estructuralista (Amate, 2007), que reivindica la necesidad de eliminar las barreras arquitectónicas como principio clave en el camino hacia la inclusión social de estas personas. Además, es digna de mención la influencia que desde los 80 hasta la actualidad, ejerce sobre estos niveles de accesibilidad, el creciente desarrollo de las Tecnologías de la Información y la Comunicación (TIC). Dicho desarrollo tecnológico constituye un nuevo modo de representación cultural que incide directamente en las maneras de entender y generar la realidad (Aguiar y Farray, 2002).

Haciendo ahora un recuento general de la presencia de los modelos por cada año y periódico estudiado, y tomando como unidades los distintos bloques categoriales de cada espacio informativo, nos percatamos de la todavía amplia conquista del modelo tradicional en 1978 y en 1995, acaparando la mitad o más de la mitad de la muestra. Ello viene a corroborar que todas las teorías avaladoras de este modelo, cuyo apogeo se mantuvo hasta finales del siglo XVII (Goodey y Stainton, 2003), siguen teniendo un cierto eco aún en nuestros días.

En cambio, la presencia del modelo rehabilitador es moderada, ya que ronda casi siempre el $20 \%$ del total por año. El único año que sobrepasa esas cifras es el 95 del diario $A B C$, aglutinando casi al $30 \%$ de la muestra. Por tanto, la contundencia del postulado rehabilitador también se hace notar en los datos recabados, pero de forma menos llamativa que en el caso anterior. Esto concuerda con las afirmaciones expuestas por autores como Schalock (2003), pues este modelo, al que él denomina "biosocial", fue dominante sólo hasta principios del siglo XX. A partir de entonces, siguió existiendo, pero de manera minoritaria, tal y como demuestran nuestros hallazgos.

Finalmente, resaltamos la escasa repercusión que todavía presenta el modelo social, despuntando con claridad sólo en el $A B C$ de 2007.

Siguiendo la línea teórica marcada por autores como Palacios y Romañach (2006); éste ha de ser el modelo de referencia en nuestros días para el fomento de la diversidad. Sin embargo, a la luz de los datos recabados, los espacios informativos desplegados sobre discapacidad en la mayoría de los casos, no se guían por esa directriz.

Ante esto, investigadores como Bueno Abad (1996), Bourdieu (1996), Trowler (1996) o Martín Herrera (2006), advierten sobre la imperiosa necesidad de cuidar la información dada a través de los medios, pues irrumpen directamente en el tejido social, provocando su transformación y cambio (Jaúregui Bereciartu, 1990). Estos cambios influyen directamente en los valores (Tajfel, 1981). Y de ahí que los medios de comunicación se hayan convertido en la base de la actual sociedad de consumo (Aguaded, 2003).

Debido a ello, se hace obligatorio profundizar con urgencia en las líneas de mejora para el tratamiento informativo apuntadas por Villanueva et al. (2006). Asimismo, estas líneas precursoras de futuros adelantos, deberán desarrollarse en paralelo con las pautas de estilo promulgadas desde documentos como los recopilados por Navarro Ruíz (2004), destacando entre ellos el de "Mejorando las comunicaciones sobre las personas impedidas", la "Declaración de Madrid" o la "Declaración de Atenas". Aparte de estos informes, despuntan en este sentido el artículo "Medios de comunicación y discapacidad" (Agencia de Noticias por los Derechos de la Infancia, 2003); 
el decálogo de consejos estipulado por Villanueva et al. (2006); los requisitos de Martín Herrera (2006) y las sugerencias de Díaz Aledo (2006).

A pesar de lo anterior, aunque prevalece el "discurso histórico de la discapacidad", hay que aclarar que poco a poco el "discurso emergente" va teniendo cabida en el panorama mediático (Benavides, 2009). Es posible que haya contribuido a esto los informes de la Clasificación Internacional de las Deficiencias, Discapacidades y Minusvalías, la Clasificación Internacional del Funcionamiento, de la Discapacidad y de la Salud, la Ley 13/1982 de 7 de Abril, de Integración Social de los Minusválidos, la Ley 51/2003, de 2 de diciembre, de Igualdad de Oportunidades, No Discriminación y Accesibilidad Universal de las personas con discapacidad, la "Carta para los años 80" o el "Programa de Acción Mundial para las Personas con Discapacidad", entre otros. Todos ellos sin duda, han contribuido y contribuyen a que el grupo social con discapacidad se vaya haciendo paulatinamente tan visible en la prensa impresa como cualquier otro.

\section{Conclusiones}

En este trabajo, hemos agrupado las unidades referenciales en torno a los tres modelos por antonomasia que se postulan ante la discapacidad: modelo tradicional, modelo rehabilitador y modelo social.

Los datos recabados sobre el modelo tradicional de la discapacidad, denotan la posición hegemónica que adoptan las referencias hacia su exaltación como algo trágico, cargante para la familia y difícil de integrar dentro del entorno social. Por su parte, bajo el modelo rehabilitador, se remarca sobre todo la necesidad de aislar y atender a estas personas en lugares ideales.

Dirigiendo ahora la mirada hacia los resultados provenientes del modelo social, lideran claramente en $A B C$ las referencias hacia las acciones que lleva a cabo la sociedad para que la situación actual de este colectivo cambie (dotación de ayudas económicas, creación de asociaciones,...). Por el lado de La Vanguardia, las referencias más destacadas durante 1978 y 2007, son aquellas que apuntan a la necesidad que demanda el colectivo de hacer valer sus derechos; mientras que en 1995, despuntan las alusiones a la creación y funcionamiento de entornos accesibles.

Finalmente, aglutinando de manera global los resultados de los tres modelos, nos damos cuenta de la todavía amplia conquista del modelo tradicional en 1978 y en 1995, acaparando la mitad o más de la mitad de las referencias. La influencia del modelo rehabilitador, en cambio, es moderada. Y la repercusión del modelo social sigue siendo escasa, despuntando con claridad sólo en el $A B C$ de 2007.

En síntesis, aunque se ha incrementado levemente el número de alusiones hacia el modelo social de la discapacidad, posiblemente gracias a los cambios normativos y terminológicos acaecidos en la última treintena, lo cierto es que aún queda un largo camino por recorrer para que la sociedad perciba que la discapacidad vivencia limitaciones importantes no tanto generadas por sus propias características, sino por las muchas restricciones contextuales que el resto de las personas le va proporcionando. La prensa tiene un potencial privilegiado para avanzar en esta línea. 


\section{Referencias bibliográficas}

AGENCIA DE NOTICIAS POR LOS DERECHOS DE LA INFANCIA (2003): $\mathrm{Me-}$ dios de comunicación y discapacidad. Brasil, Programa Regional para América Latina y El Caribe [en línea]. [Consulta: 4 de junio de 2012]. http://www.redandi.org/sites/default/files/midia_deficiencia_esp_0.pdf

AGUADED, José Ignacio (2003): "Educar en una sociedad audiovisual". En AGUIAR, María Victoria y FARRAY, Josefa (Coords.): Combyte 2003. Sociedad de la información y cultura mediática (pp. 45-63). La Coruña, Netbiblo S. L.

AGUADO DÍAZ, Antonio León y ALCEDO RODRÍGUEZ, Ma Ángeles (1991): "Tratamiento de la discapacidad en la prensa asturiana". En AA. VV.: Psicothema, 3. (pp. 175-198) [en línea]. [Consulta: 20 de marzo de 2011]. http://www.psicothema.com/psicothema.asp?id $=2013$

AGUIAR, María Victoria y FARRAY, Josefa (2007): Nuevas tecnologías aplicadas a la educación. Las Palmas de Gran Canaria, ULPGC.

AKOUN, André (1998): Sociologie des communications de masse. Paris, Hachette.

AMATE, Alicia (2007): Evolución del concepto de discapacidad [en línea]. [Consulta: 9 de julio de 2008]. http://www.larepublica.com.uy/comunidad/283129-evolucion-del-concepto-de-discapacidad-1-parte

ANDRÉU, Jaime (2000): Las técnicas de Análisis de Contenido: Una revisión actualizada. Granada: Universidad de Granada [en línea]. [Consulta: 4 de agosto de 2011]. http://public.centrodeestudiosandaluces.es/pdfs/S200103.pdf

AUSLANDER, Gail \& GOLD, Nora (1999): "Disability terminology in the media: a comparison of newspaper reports in Canada and Israel". En ANNANDALE, Ellen (Ed.): Social Science and Medicine, 48 (pp. 1395-1405). Reino Unido, Board.

BATANAZ, Luis (2000): "Legislación española sobre educación especial: un estudio introductorio". En BATANAZ, Luis y MARTÍNEZ, Rosario (Coords.): Minusvalía y educación. El valor de la diversidad, pp. 171-216. Córdoba, Servicio de Publicaciones de la Universidad de Córdoba.

BENAVIDES, Juan (2009): "Medios y discapacidad. La presencia de la discapacidad en los medios de comunicación". En AA. VV.: Revista TELOS [en línea]. [Consulta: 15 de julio de 2011]. http://sociedadinformacion.fundacion.telefonica .com/telos/experienciasgrabar.asp @idarticulo=1\&rev=62.htm

BOURDIEU, Pierre (1996): Sur la télévision. Paris, Liber.

BUENO ABAD, José Ramón (1996): Estudio longitudinal de la presencia de la mujer en los medios de comunicación de prensa escrita. Valencia, Nau llibres.

CATLETT, Susan \& MARTIN, Sylvia (1993): "Newspaper images and messages: People with disabilities". En AA. VV.: The Anual Metting of the American Association on Mental Retardation. (pp. 2-19). Texas, University of Houston.

CEA D'ANCONA, Ma Ángeles (1996): Metodología cuantitativa. Estrategias y técnicas de investigación social. Madrid, Síntesis. 
COHEN, Jacob (1960): "A coefficient of agreement for nominal scales". En AA. VV.: Educational. An Psychological Measurement, 20 (pp. 37-46). New York, New York University.

DEL RÍO, Pablo (1986): La imagen de las personas con deficiencias y el papel de los medios de comunicación. Madrid, Real Patronato sobre Discapacidad.

DÍAZ ALEDO, Loles (2006): "La representación de la discapacidad en los medios de comunicación. Cómo lograr una presencia más adecuada". En AA. VV.: Comunicación e Cidadanía, 2. [en línea]. [Consulta: 14 de julio de 2011]. http://www.observatoriodosmedios.org/mediateca/observatorio/xornadas/200611/comunicacions/artigo23.pdf

EGEA, Carlos; MIRAS, Silverio; y RIPOLL, Antonio (1995): "La imagen de las personas con discapacidad en la prensa en la región de Murcia". En AA. VV.: Intervención psicosocial IV, 11. (pp. 65-94). Madrid, Colegio Oficial de Psicólogos de Madrid.

GOODEY, Christopher \& STAINTON, Tim (2001): "Intellectual Disability and the Myth of the Changeling". En NICHOLSON, Ian (Ed.): Journal of the History of the Behavioral Sciences, 37 (pp. 223-240). Canadá, St. Thomas University.

JAÚREGUI BERECIARTU, Gurutz (1990): "Medios de información y poder: el control de los flujos de información". En AA. VV.: Revista Española de Investigaciones Sociológicas, 49 (pp. 91-115). Madrid, CIS.

JIMÉNEZ LARA, Antonio (1999): La imagen social de la discapacidad [en línea] [Consulta: 13 de julio de 2011]. http://usuarios.discapnet.es/AJimenez/imagen/ imagensocial.htm

KELLER, Clayton; HALLAHAN, Daniel; MCSHARE, Edward; CROWLEY, Paula; \& BLANDFORD, Barbara (1990): "The coverage of persons with disabilities in American newspapers". En AA. VV.: The journal of special education, 3. (pp. 271282). USA, University of North Carolina at Charlotte.

KRIPPENDORFF, Klaus (1990): Metodología de análisis de contenido. Teoría y práctica. Barcelona: Paidós Ibérica.

MARCHENA, Rosa; GARRIDO, Jesús; y LAJUSTICIA, Fernando (2007): ¿Todo sobre ruedas? Rendimiento laboral sostenible: el empleo de personas con discapacidad. Las Palmas de Gran Canaria, Fundación Canaria ICSE.

MARTÍN HERRERA, Inma (2006): "El papel de los medios de comunicación en la imagen social de la discapacidad". En AA. VV.: Comunicación e Cidadanía, 2 [en línea]. [Consulta: 13 de abril de 2008]. http://observatoriodosmedios.org/mediateca/observatorio/ xornadas/200611/comunicacions/artigo3.pdf

NAVARRO RUÍZ, Cristina (Dir., 2004): La imagen de la discapacidad en la prensa de Castilla y León. Salamanca: Europa Artes gráficas.

PALACIOS, Agustina y ROMAÑACH, Javier (2006): El modelo de la diversidad. La Bioética y los Derechos Humanos como herramientas para alcanzar la plena dignidad en la diversidad funcional. Madrid, Diversitas. 
SCHALOCK, Robert (2003): "El paradigma emergente de la discapacidad y sus retos en este campo". En VERDUGO, Miguel Ángel y JORDÁN DE URRIES, Borja (Coords.): Investigación, Innovación y cambio. Actas de las V Jornadas cientificas de investigación sobre personas con discapacidad. (pp. 193-217). Salamanca, Amarú.

TAJFEL, Henri (1981): Human Groups and Social Categories. Cambridge,:Cambridge University Press.USA, Collins Educational.

VEGA, Amando y MARTÍN, Raquel (1999): "Los medios de comunicación ante la discapacidad: más allá de los estereotipos". En AGUADED, José Ignacio (Ed.): Comunicar, 12. (pp. 111-116) [en línea]. [Consulta: 13 de abril de 2008]. http://redalyc.uaemex.mx/ redalyc/pdf/158/15801217.pdf

VILLANUEVA, José; BARBERENA, Tareixa; y REBOIRAS, Óscar (2006): "Los medios de comunicación como integradores de las personas con discapacidad: realidad gallega y medidas a tomar". En AA. VV.: Maremagnum, 10, pp. 151-160. Santiago de Compostela, Autismo Galicia.

WEINBERG, Nancy \& WILLIAMS, Judy (1998): "How the Physically Disabled Perceive their Disabilities". En AA. VV.: Journal of Rehabilitation, pp. 31-33. Chicago, National Easter Seal Society. 